\title{
Characterization of the gut microbiota in the golden takin (Budorcas taxicolor bedfordi)
}

\author{
Jun Chen ${ }^{1 \dagger}$, Huanxin Zhang ${ }^{2 \dagger}$, Xiaoyang Wu ${ }^{1+}$, Shuai Shang ${ }^{2}$, Jiakuo Yan ${ }^{1}$, Yao Chen ${ }^{1}$, Honghai Zhang ${ }^{1 *}$ \\ and Xuexi Tang ${ }^{2^{*}}$
}

\begin{abstract}
The gut microbiota of mammals is a complex ecosystem, which is essential for maintaining gut homeostasis and the host's health. The high throughput sequencing allowed us to gain a deeper insight into the bacterial structure and diversity. In order to improve the health status of the endangered golden takins, we first characterized the fecal microbiota of healthy golden takins using high throughput sequencing of the $16 \mathrm{~S}$ rRNA genes $\mathrm{V} 3-\mathrm{V} 4$ hypervariable regions. Our results showed that, Firstly, the gut microbiota community comprised 21 phyla, 40 classes, 62 orders, 96 families, and 216 genera. Firmicutes (67.59\%) was the most abundant phylum, followed by Bacteroidetes (23.57\%) and Proteobacteria (2.37\%). Secondly, the golden takin maintained higher richness in spring than in the winter while community diversity and evenness was not significantly different. Thirdly, four female golden takins demonstrated highly similar microbiota and the five golden takin males had relatively highly similar microbiota. All of our results might indicate that the fecal microbiota of golden takins were influenced by the season and the animal's sex. The findings provided theoretical basis regarding the gut microbiota of golden takins and may offer new insights to protect this endangered species.
\end{abstract}

Keywords: Gut microbiota, 16SrRNA, Illumina sequencing, Seasonal changes, Diet quality, Golden takin (B. t. bedfordi)

\section{Introduction}

The takin (Budorcas taxicolor) is a large Himalayan ungulate in the family Bovidae, which is classified into four subspecies based on distinct differences in physical characteristics and geographic location, including the golden takin (B. t.bedfordi), the Mishmi takin (B. t.taxicolor), the Sichuan takin (B.t. Tibetana), and the Bhutan takin (B. t.whitei). The golden takin and the Sichuan takin are endemic subspecies of China. Furthermore, relative to the other subspecies, the golden takin is listed as vulnerable by the International Union for Conservation of Nature (IUCN 2008) and is under state protection (category I) in China. The golden takin is a type of social bovid herbivore. As a ruminant, this animal primarily forages on the browse of trees and shrubs (Schaller et al. 1986). In the spring and summer, golden takins undertake seasonal

\footnotetext{
*Correspondence: zhanghonghai67@126.com; tangxx@ouc.edu.cn

† Jun Chen, Huanxin Zhang and Xiaoyang Wu contributed equally to this work

${ }^{1}$ College of Life Science, Qufu Normal University, Jing xuan West Street No. 57,

Qufu 273165, Shandong, People's Republic of China

${ }^{2}$ College of Marine Life Sciences, Ocean University of China, Qingdao, China
}

migration to the higher elevations of the uppermost tree line. During the autumn and winter, they move to lower elevations with smaller groups (Ming et al. 2003).

The gut microbiota offers substantial benefits to the host, such as aiding in digestion, promoting immune system development and competing for niches with pathogens (Cadwell 2014). To date, there have been many studies investigating the gut microbiota in herbivores (Bian et al. 2013; Costa et al. 2012; Liu et al. 2014). For instance, Firmicutes and Bacteroidetes, represented by the Ruminococcaceae, Lachnospiraceae, Rikenellaceae and Prevotellaceae families (Bian et al. 2013), dominate the microbiota in the healthy rhinoceros. Costa et al. (2012) described the predominance of Firmicutes (68\%) in the feces of healthy horses, followed by Bacteroidetes (14\%) and Proteobacteria (10\%). The core microbiota of donkeys is dominated by Firmicutes (64\% males and 64\% females) and Bacteroidetes (23\% males and $21 \%$ females), followed by Verrucomicrobia, Euryarchaeota, Spirochaetes and Proteobacteria (Liu et al. 2014). Considerable evidence supports the complexity of the gut microbiota in many mammals and the necessity of gut bacterial communities to maintain gut homeostasis. 
However, surprisingly, little research was on the gut microbiota of the golden takin. Therefore, in present research, we characterized the fecal microbiota of nine golden takins by performing high-throughput sequencing. On this basis, we also compared gut microbiota variation from winter to spring, which is the season of animals likely to get sick. Our result indicated that season was a factor that might impact the composition of the gut microbiota.

\section{Materials and methods}

\section{Animal selection}

Nine healthy golden takins were enrolled in this study (Table 1). Four fresh fecal samples (Group A) were collected at Jinan wild animal park during the late morning in December 2015. Five fresh fecal samples (Group B) were collected at Jinan Animal Park during the late morning in April 2016. Group A and group B respectively represented the winter and spring feces samples. All of the animals were about 8 years old and the two environments were roughly same. The experiment was approved by the Qufu Normal University Animal Care and Use Committee. None of the animals had received anti-inflammatory drugs or antimicrobials within the prior 4 months, and none had a gastrointestinal-related disease. Samples were collected off the ground immediately after defecation, and each sample was instantly transferred into plastic tubes. The samples were stored at $4{ }^{\circ} \mathrm{C}$ with long-term storage at $-80^{\circ} \mathrm{C}$ for further analysis.

\section{DNA extraction, 16S rRNA gene PCR and sequencing}

Total genomic DNA was isolated from fecal samples using a QIAamp DNA Stool Mini Kit (Qiagen, Germany) according to the manufacturer's recommended protocol. We used a UV-Vis spectrophotometer (NanoDrop 2000c, USA) to determine DNA quantification and quality. DNA concentrations were measured to ensure final concentrations greater than $20 \mathrm{ng} / \mu \mathrm{l}$ for PCR amplification of the V3-V4 hypervariable regions of the 16SrRNA gene using the primers 341F (CCTAYGGGRBGCASCAG) and 806R

Table 1 Information on the characteristics of the golden takins used in this study

\begin{tabular}{llll}
\hline Group & Golden takin & Sex & Place \\
\hline A & $J 1$ & Female & Jinan wild animal park \\
& $J 2$ & Male & Jinan wild animal park \\
& $J 3$ & Male & Jinan wild animal park \\
& $J 4$ & Male & Jinan wild animal park \\
& $J 5$ & Female & Jinan animal park \\
B & Male & Jinan animal park \\
& $J 7$ & Male & Jinan animal park \\
& J8 & Female & Jinan animal park \\
& J9 & Female & Jinan animal park \\
& & &
\end{tabular}

(GGACTACNNGGGTATCTAAT), as previously described (Hugerth et al. 2014). Amplification was performed in a $30-\mu \mathrm{l}$ volume, and the amplified fragment size was approximately $410 \mathrm{bp}$. Specifically, PCR reactions contained $15 \mu \mathrm{l}$ of Phusion Master Mix (2er), $1.5 \mu \mathrm{l}$ of each primer, $10 \mu \mathrm{l}$ of microbial genomic DNA, and $2 \mu \mathrm{ddH}_{2} \mathrm{O}$ to constitute a final volume of $30 \mu \mathrm{l}$. The following PCR protocol was used: $1 \mathrm{~min}$ at $98{ }^{\circ} \mathrm{C}$ for initial denaturation, 35 cycles of $10 \mathrm{~s}$ at $98{ }^{\circ} \mathrm{C}$ for denaturation, $30 \mathrm{~s}$ at $55^{\circ} \mathrm{C}$ for annealing, and $30 \mathrm{~s}$ at $72{ }^{\circ} \mathrm{C}$ for elongation, and a final extension at $72{ }^{\circ} \mathrm{C}$ for $5 \mathrm{~min}$. PCR products were mixed with same volume of $1 \times$ loading buffer (containing SYBR green) and separated by electrophoresis on a $2 \%$ agarose gel. Samples containing a bright band between 400 and 450 bp were chosen and mixed in equidensity ratios. Then, this mixture of PCR products was purified with a Qiagen Gel Extraction Kit (Qiagen, Germany). Sequencing libraries were generated using a TruSeq ${ }^{\circledR}$ DNA PCR-Free Sample Preparation Kit (Illumina, USA) following the manufacturer's recommendations, and index codes were added. Library quality was assessed with a Qubit@ 2.0 Fluorometer (Thermo Scientific) and an Agilent Bioanalyzer 2100 system. Finally, the DNA library was sequenced on an IlluminaHiSeq 2500 platform, and 250-bp paired-end reads were generated.

\section{Bioinformatics analysis}

Paired-end reads were assigned to samples based on their unique barcodes and truncated by cutting off the barcodes and primer sequences. Paired-end reads were then merged using FLASH (V1.2.7) (Magoč and Salzberg 2011). The raw tags were subjected to specific filtering conditions to obtain high-quality clean tags using the QIIME (V1.7.0) (Caporaso et al. 2010) quality control process. The tags were compared with the reference database (Gold database) using the UCHIME algorithm (UCHIME Algorithm) (Edgar et al. 2011) to detect chimeric sequences, which were then removed. Finally, effective tags were obtained. Sequence analysis was performed using Uparse software (Uparse v7.0.1001) (Edgar 2013). Sequences demonstrating greater than or equal to $97 \%$ similarity were assigned to the same operational taxonomic unit (OTU). For each representative sequence, the GreenGene Database (Desantis et al. 2006) was used and the RDP classifier algorithm (Version 2.2) (Wang et al. 2007) was employed to annotate taxonomic information. To study the phylogenetic relationships between different OTUs and identify dominant species in different samples (groups), multiple sequence alignment was carried out using MUSCLE software (Version 3.8.31) (Edgar 2004). Ultimately, we obtained 54,748 sequences for diversity analysis. Alpha diversity was evaluated by calculating six indices, specifically the Observed-species, Chao1, Shannon, Simpson, ACE and Good-coverage indices. All indices were calculated 
with QIIME (V1.7.0) and displayed with R software (Version 2.15.3). Beta diversity based on both Weighted and Unweighted Unifrac distances was calculated using QIIME software (Version 1.7.0). Cluster analysis was preceded by principal component analysis (PCA) using the FactoMineR package and ggplot2 package in R software (Version 2.15.3). The data set supporting the results of this article is available in the Sequencing Read Archive (SRA) database, accession numbers SRP090712.

\section{Results}

During quality control, a total of 670,356 high-quality sequences with an average length of $410 \mathrm{bp}$ per sample were obtained from the nine fecal samples. The statistical estimates of species richness for the sequences from the nine samples that demonstrated a genetic distance of $3 \%$, the total number of sequences, the coverage, and the number of OTUs were shown in Table 2. The rarefaction curves and rank abundance curves were generated using R software (Version 2.15.3). The rarefaction curves tended to approach the saturation plateau, while the rank abundance curves revealed a few dominant taxa and many low-abundance taxa comprising the bacterial community of the golden takin (Fig. 1). Based on ANOSIM analysis, significant differences were observed in bacterial community structure between group A and group B $(P<0.05)$. When analyzing differences between the two groups, the Alpha diversity index and box-plots intuitively reflected the median, degree of dispersion, maximum, minimum and outlier of species diversity within

Table 2 Alpha-diversity of the gut microbiota in the nine golden takins in this study

\begin{tabular}{|c|c|c|c|c|c|c|}
\hline Sample & Observed species & Shannon & Simpson & Chao1 & ACE & Goods coverage \\
\hline J1 & 1271 & 8.309 & 0.991 & 1342.163 & 1343.512 & 0.998 \\
\hline$J 2$ & 1292 & 8.203 & 0.992 & 1358.65 & 1399.993 & 0.997 \\
\hline $\mathrm{J3}$ & 1299 & 8.276 & 0.991 & 1373.654 & 1375.156 & 0.997 \\
\hline$J 4$ & 1300 & 8.26 & 0.991 & 1394.565 & 1408.729 & 0.997 \\
\hline$J 5$ & 1366 & 8.394 & 0.992 & 1444.019 & 1456.449 & 0.997 \\
\hline$J 6$ & 1495 & 8.634 & 0.993 & 1566.16 & 1582.534 & 0.997 \\
\hline$J 7$ & 1450 & 8.644 & 0.994 & 1532.564 & 1541.698 & 0.997 \\
\hline J8 & 1405 & 8.428 & 0.992 & 1542.244 & 1520.494 & 0.996 \\
\hline 19 & 1289 & 8.1 & 0.989 & 1355.071 & 1360.503 & 0.997 \\
\hline
\end{tabular}
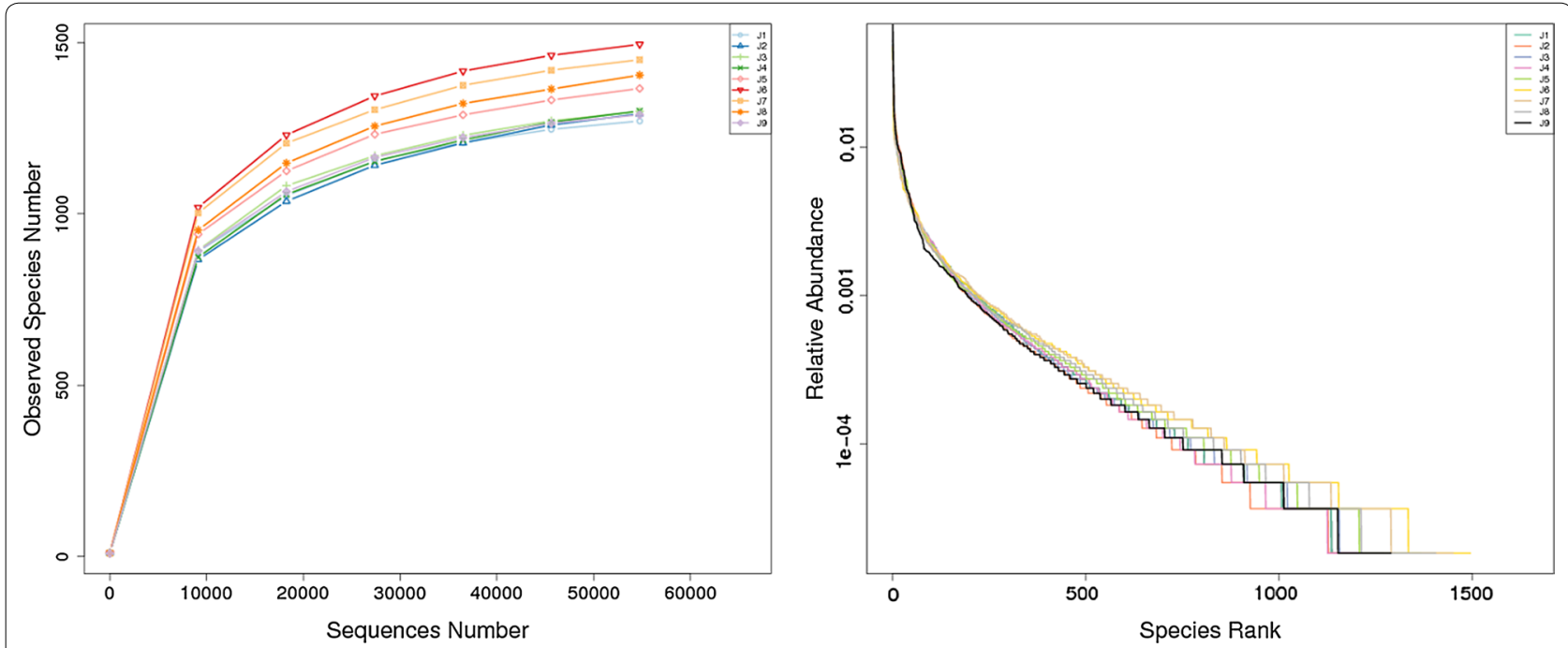

Fig. 1 Rarefaction curve analysis of nine golden takins. Repeated samples of OTU subsets were performed to evaluate whether further sampling would likely yield additional taxa, as indicated by whether the curve reached a plateau value. The $y$-axis indicates the number of OTUs detected, and the $x$-axis indicates the number of taxa in the sequence subset analyzed. Rank abundance curves were used to estimate the richness and evenness of taxa present in the samples. The $y$-axis indicates the relative abundance of OTUs, and the $x$-axis indicates the number of OTUs according to the relative abundance from large to small. The larger the span curve on the $x$-axis, the higher the species richness. The smoother the curve on the $y$-axis, the more even the species distribution 
groups. The number of species in group A was lower than that in group B (Fig. 2), and this difference was significant $(P<0.05)$. However the difference of the community diversity and the species distribution uniformity between two groups were not significant $(P>0.05)$.

\section{Taxonomic composition}

A total of 20 prokaryotic phyla were identified from the nine golden takins (Fig. 3a). The majority of their gut flora belonged to two phyla: Firmicutes (66.3769.23\%) and Bacteroidetes (21.26-26.55\%). These two phyla represented more than $90 \%$ of the total sequences in all nine samples. However, Euryarchaeota belonged to the Archaeal domain, and the remainder of the identified bacteria belonged to the bacterial domain. Furthermore, variations were noted in the microbiota among samples, e.g. Synergistetes was only found in samples J1, J6, J7, and J8; Fusobacteria was only identified in samples J6 and J7; Gemmatimonadetes was only observed in sample J7; Deferribacteres were identified in sample J6; Chloroflexi was only present in $\mathrm{J} 1, \mathrm{~J} 5$, and $\mathrm{J} 8$.

At the family level, we detected 96 families, and the abundance of unclassified bacteria in the samples was $17.04 \%$. The distribution proportion was greater than $0.5 \%$ for 10 families (Fig. 3b). Among these 10 families, Ruminococcaceae was predominant, with an abundance of $49.61 \%$, followed by Rikenellaceae (10.93\%), Lachnospiraceae (6.41\%), Christensenellaceae (3.68\%), Clostridiales_vadinBB60_group (3.15\%), Prevotellaceae (2.92\%), Bacteroidaceae (2.64\%), Bacteroidales_RF16_group (2.32\%), Verrucomicrobiaceae (0.79\%), and Bacillaceae
(0.50\%). The most abundant families were Ruminococcaceae, Rikenellaceae and Lachnospiraceae, which comprised approximately $66.95 \%$ of total sequences.

At the genus level, the percentage of unclassified bacteria averaged $42.52 \%$ (ranging from 36.7 to $50.27 \%$ ), and the remainder were classified into 216 genera. The distribution proportion was greater than $0.5 \%$ in 10 genera (Fig. 3c). Among these 10 genera, Ruminococcaceae_UCG-010 was predominant, with an abundance of $21.44 \%$, followed by Ruminococcaceae_UCG-005 (13.93\%), Rikenellaceae_ RC9_gut_group (7.30\%), Christensenellaceae_R-7_group (3.56\%), Alistipes (2.84\%), Bacteroides (2.64\%), [Eubacterium]_coprostanoligenes_group (2.36\%), Ruminococcaceae_UCG-013 (2.11\%), Akkermansia (0.79\%), and Bacillus (0.50\%). Of the 10 genera, Ruminococcaceae_UCG-010, Ruminococcaceae_UCG-005, Christensenellaceae_R-7_group, Ruminococcaceae_UCG013, Bacillus, and [Eubacterium]_coprostanoligenes_group belong to Firmicutes. Rikenellaceae_RC9_gut group, Alistipes and Bacteroides belong to Bacteroidetes, and Akkermansia belongs to Verrucomicrobia.

To know the bacterial community profiles at the genus level, we plotted a clustered heatmap. The result indicated that the sample J1, J2, J3 and J4 (group A) grouped together, while the sample J5, J6, J7 and J8 (group B) clustered together (Fig. 4). To compare the microbial communities across the different samples, we plotted another heatmap based on Weighted Unifrac and Unweighted Unifrac distances (Fig. 5). The similarity of the samples was consistent with the clustering results. In addition, the PCA score plot revealed the separation of group A from group B (Fig. 6), which was consistent with the heatmap results.
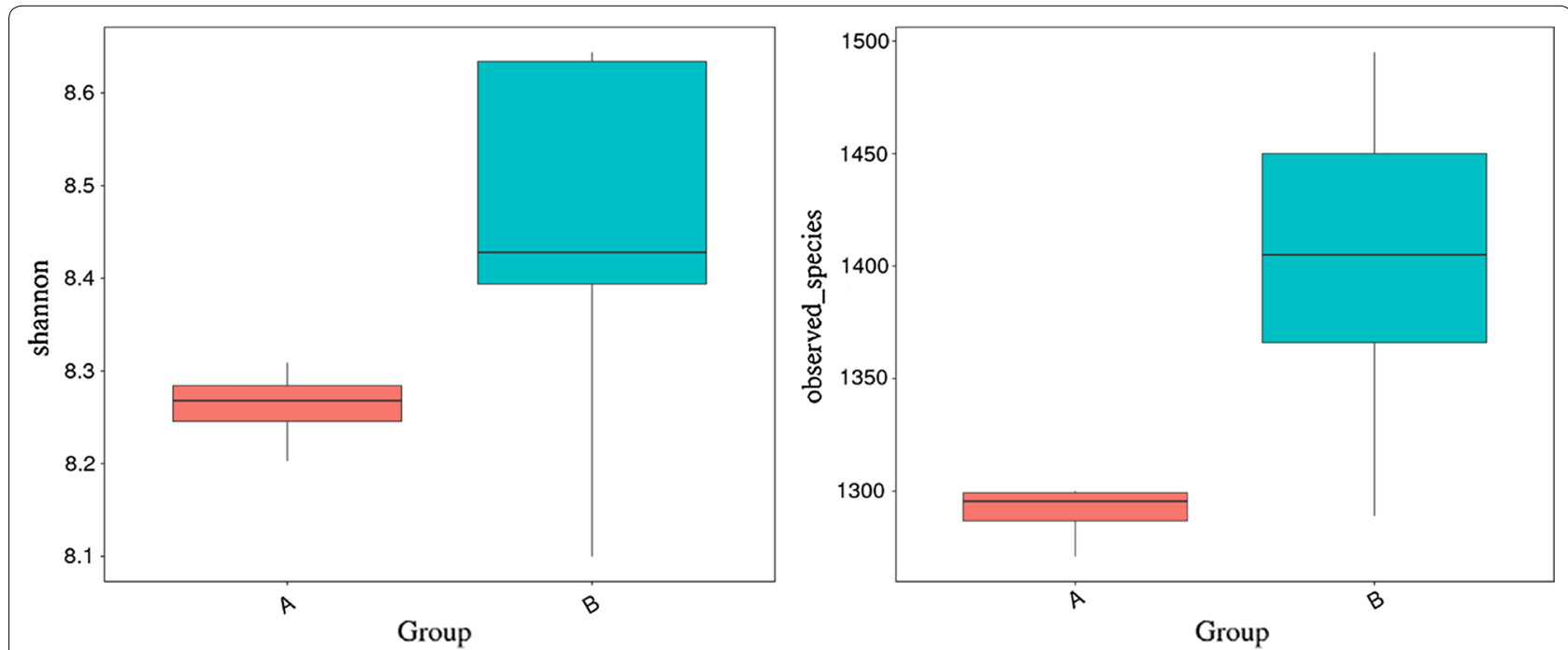

Fig. 2 A comparison of community alpha diversities between winter and spring samples in golden takins. Diversity was measured by calculating the Shannon index, and richness was determined by calculating the Observed-species index 


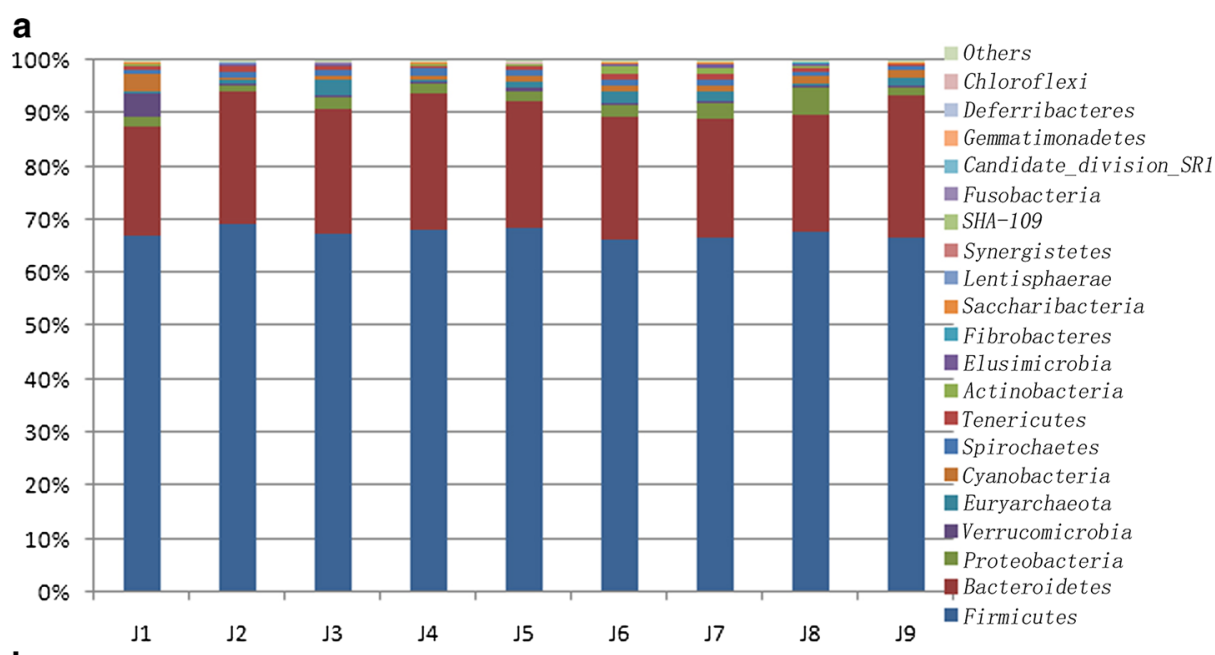

b
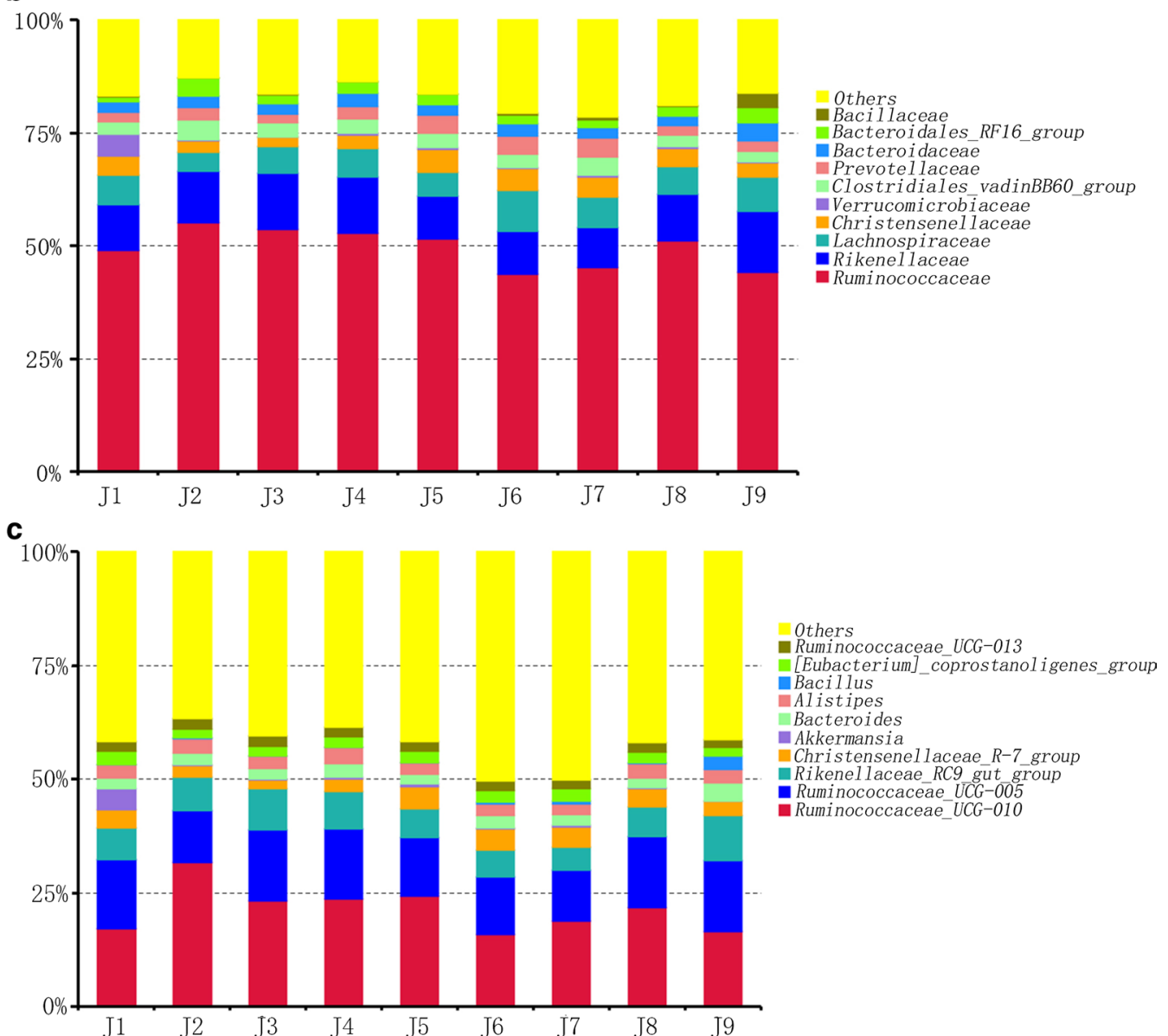

Fig. 3 Gut bacterial composition at the phylum level per sample (a) and the relative abundance of the top ten biological species at the family level (b) and the genus level (c)

\section{Analysis of species abundance}

We performed $t$ test and Mann-Whitney $U$ test to evaluate the significant differences $(P<0.05)$ among species. At the phylum level, Actinobacteria was significantly more abundant in group B than in group A. At the class level, Coriobacteriia and Deltaproteobacteria were significantly less abundant in group A than in group B. At the order level, Coriobacteriales was also significantly 


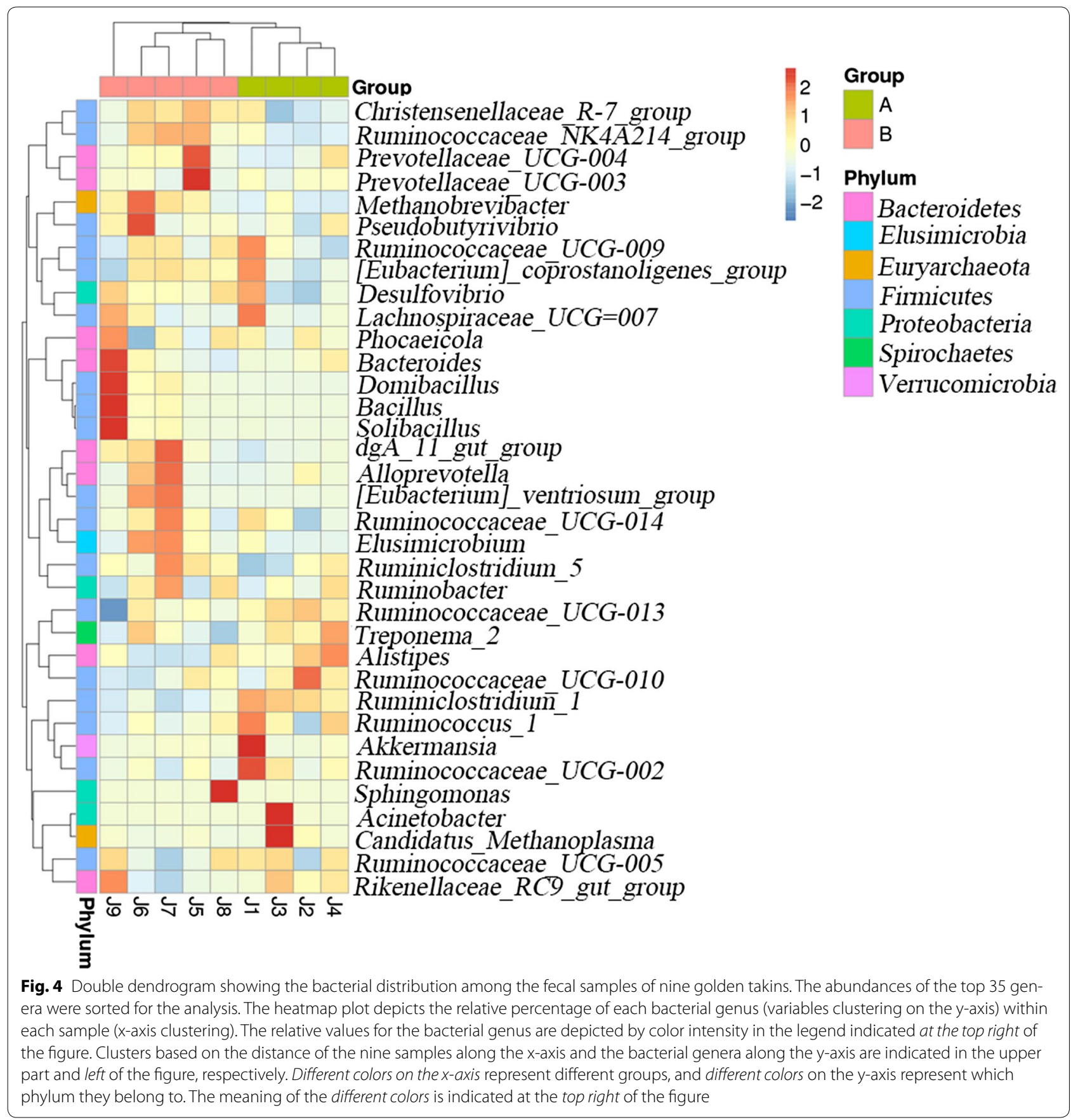

higher in group B. At the family level, Coriobacteriaceae was significantly higher in group B, while Ruminococcaceae and Bacteroidales_S24-7_group were higher in group A (Table 3). Eighteen of the 216 genera were significantly different $(P<0.05)$ between group $\mathrm{A}$ and group B (Table 4). Among these 18 genera, Parabacteroides, Sphaerochaeta, Ruminiclostridium_1 and Phascolarctobacterium were significantly higher in group A, while the others were significantly higher in group B. Additionally, 13 of the 18 genera belonged to Firmicutes.

\section{Discussion}

In present study, we first performed high-throughput sequencing to characterize the microbial community in the golden takin. Similar to previous researches (Costa et al. 2012; Oikonomou et al. 2013), the golden takin gut 


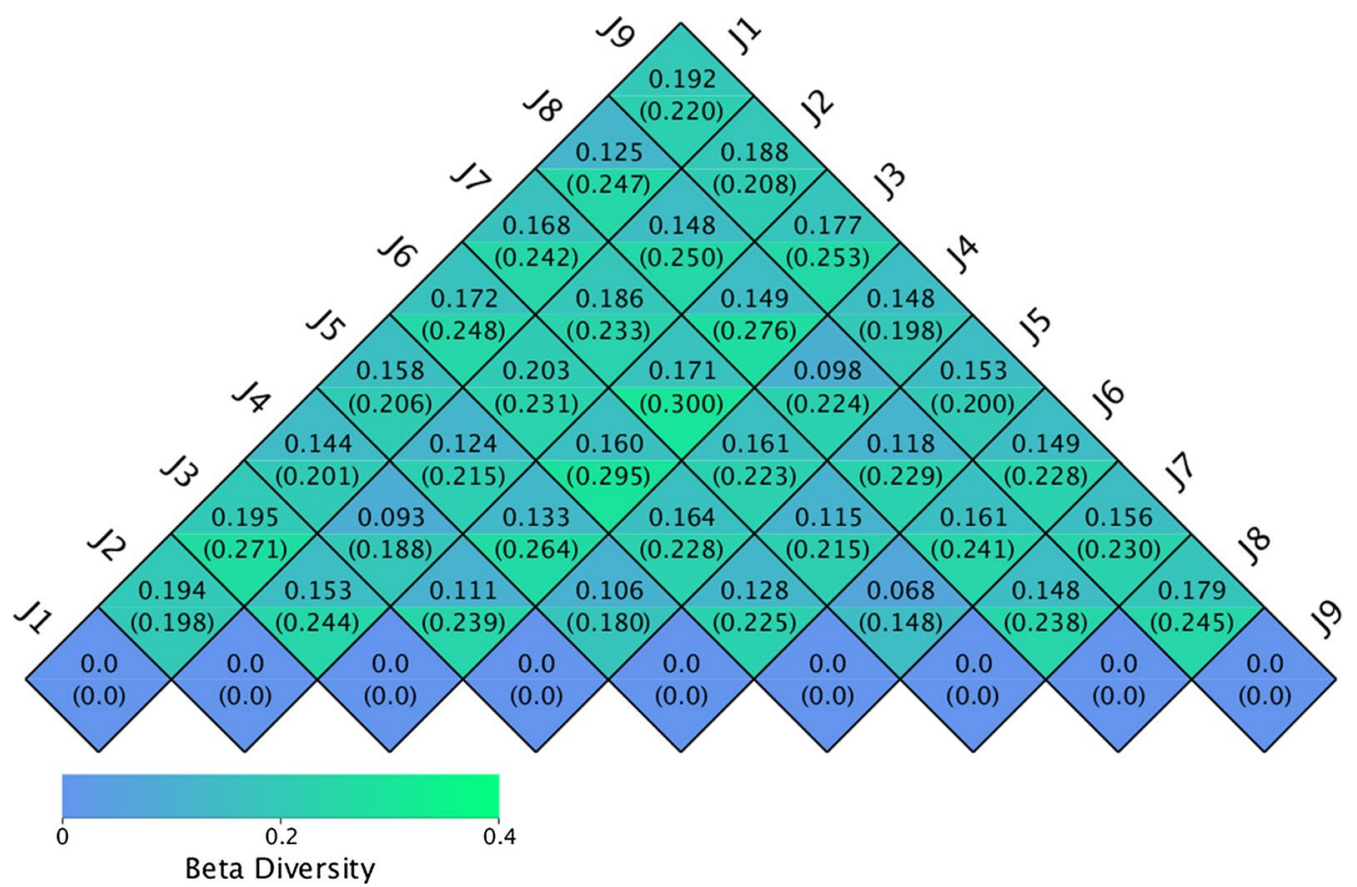

Fig. 5 Heatmap of Weighted Unifrac and Unweighted Unifrac distances. In the same square, a fluctuation in two values indicates the Weighted Unifrac and Unweighted Unifrac distances. The number in the diagram pane is the discrepancy coefficient between any two samples. The smaller the discrepancy coefficient between two samples, the smaller the difference in species diversity

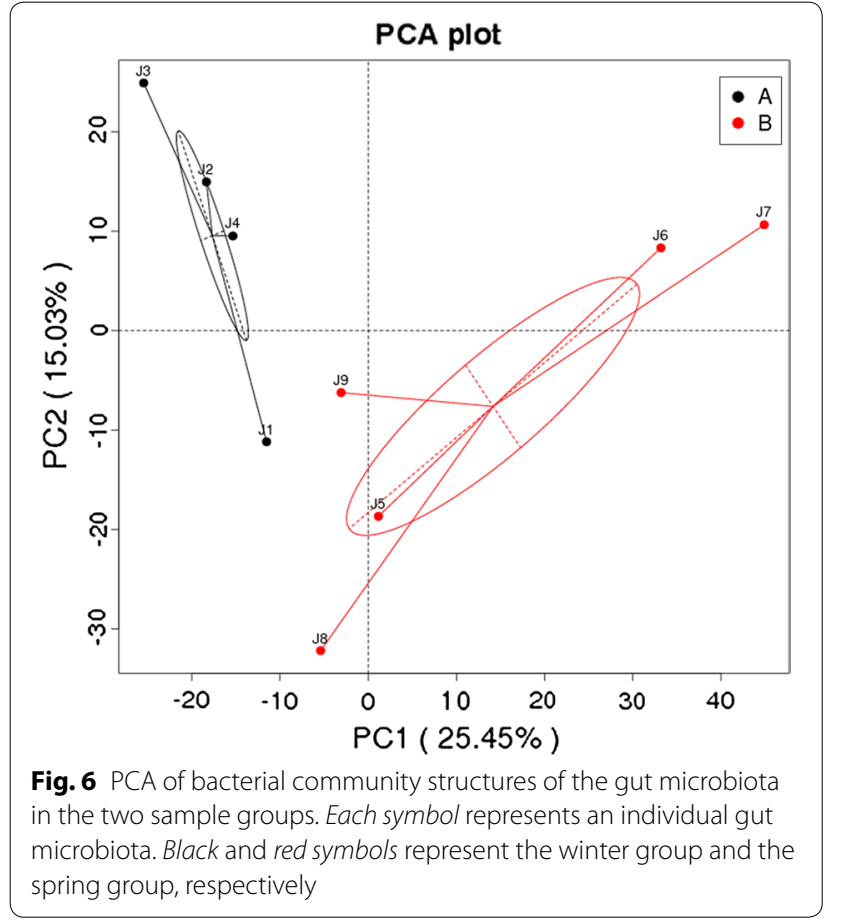

microbiota was also dominated by Firmicutes (67.59\%) and Bacteroidetes (23.57\%), followed by Proteobacteria (2.37\%). Firstly, Firmicutes was an advantaged group
Table 3 Seasonal (winter and spring) comparisons for species abundance at the phylum, class, order and family levels

\begin{tabular}{lcccl}
\hline Taxa & Avg (A) (\%) & Avg (B) (\%) & P value & Annotation \\
\hline Actinobacteria & 0.13 & 0.73 & 0.016 & Phylum \\
Coriobacteriia & 0.11 & 0.66 & 0.016 & Class \\
Deltaproteobacteria & 0.63 & 0.88 & 0.041 & Class \\
Coriobacteriales & 0.11 & 0.66 & 0.016 & Order \\
Ruminococcaceae & 52.67 & 47.17 & 0.039 & Family \\
$\begin{array}{l}\text { Bacteroidales_S24- } \\
\quad \text { 7_group }\end{array}$ & 0.99 & 0.33 & 0.016 & Family \\
Coriobacteriaceae & 0.11 & 0.66 & 0.016 & Family \\
p-2534-18B5_gut_ & 0.30 & 0.75 & 0.012 & Family \\
group & & & & \\
\hline
\end{tabular}

of bacteria in the gastrointestinal tract and fecal flora of various animals (Ley et al. 2008). The advantage exhibited by Firmicutes might be related to the anatomical physiology and diet of specific species that feed primarily upon insoluble fiber and in which the cecum and colon were the major sites of fermentation (Costa et al. 2012). Within Firmicutes, Clostridiales (65.42\%) dominated in the feces of golden takins. According to previous studies, Clostridiales accounted for $24-59 \%$ of human fecal samples and was also the dominant order in the canine 
Table 4 Seasonal (winter and spring) comparisons for species abundance at the genus level

\begin{tabular}{|c|c|c|c|c|}
\hline Phylum & Genus & $\operatorname{Avg}(A)(\%)$ & Avg (B) (\%) & $P$ value \\
\hline Euryarchaeota & $\begin{array}{l}\text { Methanobrevi- } \\
\text { bacter }\end{array}$ & 0.239 & 1.008 & 0.049 \\
\hline Actinobacteria & Atopobium & 0.046 & 0.267 & 0.032 \\
\hline Actinobacteria & Senegalimassilia & 0.012 & 0.076 & 0.019 \\
\hline Bacteroidetes & Parabacteroides & 0.050 & 0.024 & 0.012 \\
\hline Spirochaetes & Sphaerochaeta & 0.025 & 0.007 & 0.007 \\
\hline Firmicutes & $\begin{array}{l}\text { Ruminococcaceae } \\
\text { NK4A214_group }\end{array}$ & 0.513 & 0.765 & 0.033 \\
\hline Firmicutes & $\begin{array}{l}\text { Ruminiclostrid- } \\
\text { ium_1 }\end{array}$ & 0.765 & 0.366 & 0.001 \\
\hline Firmicutes & Ruminococcus_2 & 0.168 & 0.397 & 0.000 \\
\hline Firmicutes & $\begin{array}{l}\text { Phascolarctobac- } \\
\text { terium }\end{array}$ & 0.367 & 0.267 & 0.047 \\
\hline Firmicutes & Acetitomaculum & 0.030 & 0.101 & 0.016 \\
\hline Firmicutes & $\begin{array}{l}\text { Hydrogenoanaero- } \\
\text { bacterium }\end{array}$ & 0.093 & 0.119 & 0.046 \\
\hline Firmicutes & $\begin{array}{l}\text { [Eubacterium]_ } \\
\text { nodatum_group }\end{array}$ & 0.046 & 0.102 & 0.011 \\
\hline Firmicute & Marvinbryantia & 0.041 & 0.076 & 0.032 \\
\hline Firmicutes & $\begin{array}{l}\text { Lachnospiraceae } \\
\text { UCG-001 }\end{array}$ & 0.030 & 0.070 & 0.005 \\
\hline Firmicutes & $\begin{array}{l}\text { Lachnospiraceae } \\
\text { NK4B4_group }\end{array}$ & 0.025 & 0.046 & 0.009 \\
\hline Firmicutes & Solobacterium & 0.005 & 0.021 & 0.036 \\
\hline Firmicutes & $\begin{array}{l}\text { Family_XIII_UCG- } \\
001\end{array}$ & 0.011 & 0.022 & 0.024 \\
\hline Firmicutes & Papillibacter & 0.005 & 0.014 & 0.035 \\
\hline
\end{tabular}

gut microbiota; thus, this family was an important index of intestinal bacterial ecosystem function and metabolic differences (Wang et al. 2005). Additionally, Ruminococcaceae dominated in the nine golden takins, and Lachnospiraceae was present at elevated levels; these bacteria were regarded as fiber-degraders in the herbivore rumen and hindgut (Bian et al. 2013; Jami and Mizrahi 2012). Trace amounts of another major fiber-degrader in the rumen, Fibrobacter, were detected in the feces of the nine golden takins. Secondly, Bacteroidetes were the secondmost predominant components of the gut flora in the nine golden takins, which were consistent with previous researches (Bian et al. 2013; Costa et al. 2012; Oikonomou et al. 2013). Conversely, Bacteroidetes constitutes the dominant group in the rumens of other ruminants (Bhatt et al. 2013; Jami and Mizrahi 2012). Bacteroidetes degraded high-molecular-weight organic matter, such as protein and carbohydrates, and complemented the host genome to degrade resistant foods such as plant cell wall compounds (including cellulose, pectin, and xylan) (Thoetkiattikul et al. 2013). Bacteroides also degraded host sources of carbohydrates, primarily derived from gastrointestinal tract secretions, such as mucins and chondroitin sulfate polysaccharides (Salyers et al. 1977). Additionally, Bacteroidetes produces butyrate, which was the product of colonic fermentation. Bacteroidetes possessed antitumor properties, which played important roles in maintaining intestinal health (Kim and Milner 2007), and participated in toxic or mutagenic bile acid metabolism and transformation (Smith et al. 2005). In our study, the families Rikenellaceae, Prevotellaceae and Bacteroidaceae dominated within this phylum. Thus, Bacteroidetes played an equally important role as Firmicutes in the digestive system of herbivorous animals. Thirdly, Proteobacteria constituted a large group of bacteria comprising various bacterial taxa that are capable of catabolizing a wide range of feedstuff components (Evans et al. 2010), which advantages these bacteria in the panda (Wei et al. 2012). These differences may be caused by different community structures in the host diet and bowel. In our study, at the genus level, the highest species distribution proportions were observed for Ruminococcaceae_UCG-010 and Ruminococcaceae_UCG-00, which belonged to Ruminococcaceae and were involved in fiber digestion. Fourthly, unclassified bacteria in the nine samples accounted for $18.82-22.48 \%$ of sequences. According to previous studies, many animal gut microbiomes contained certain proportions of unclassified bacteria (Bian et al. 2013; Costa et al. 2012; Wu et al. 2016). The reason of this might due to the limited database of $16 \mathrm{~S}$ RNA gene sequences and little research on classification of fecal microbes. Unclassified bacteria and their functions in different hosts required further research.

The PCA and heatmap analyses indicated that the separation was observed between the microbiota of group A and group B. However, within groups, there was a high percent identity, suggesting the fecal microbiota of golden takins may be influenced by different seasons. Moreover, rarefaction curves revealed lower phylogenetic diversity and numbers of observed species (OTUs) in the winter microbiota than in the spring microbiota. At the same time, the spring microbiota exhibited higher numbers of OTUs with significant differences (Fig. 2). Sun et al. (2016) also found that the dietary shift from winter to spring affected the Tibetan macaque gut microbial composition and diversity. For our research, the diets of the nine golden takins in winter and spring were roughly the same. The significant differences $(P<0.05)$ among species showed that the relative abundances of Firmicutes and Bacteroidetes in spring were slightly decreased compared with those in winter, Acidobacteria differed significantly between the two seasons and was significantly higher in the spring at the phylum level. According to previous studies, Acidobacteria was present in many wetland soils and contained a number of genes 
encoding cellulases and hemicellulases. Thus, these bacteria might play an important roles in plant residue degradation (Kanokratana et al. 2011; Pankratov et al. 2011). At the class level, Coriobacteriia and Deltaproteobacteria were significantly more abundant in the spring than in the winter. Coriobacteriia belonged to Actinobacteria, and Deltaproteobacteria belonged to Proteobacteria. Actinobacteria was an important secondary metabolite (enzyme and antibiotics) producer that also played this role in animal intestines, and the secondary metabolites of Actinobacteria are potent antibiotics (Bull et al. 2005; Cundliffe 2006). We hypothesized that the rise in Coriobacteriia facilitated better resistance to harmful microorganisms external to the intestinal environment, and the rise in Deltaproteobacteria was attributable to decreased food quality. Instestingly, 18 genera were significantly different $(P<0.05)$ between spring and winter, and 13 of these 18 genera belonged to Firmicutes; among these 13 genera, two genera exhibited higher abundances in the winter than in the spring. In contrast, the other 11 genera were less abundant in the winter than in the spring. Thus, Firmicutes might play different roles in different seasons (Carey et al. 2012; Ley et al. 2008; Taglialatela et al. 2009). The weather in spring was highly variable, which was suitable for the proliferation of a variety of pathogenic microorganisms; thus, food might contain greater numbers of pathogenic microorganisms. We suspected that the seasonal differences might be due to the feeding quality, and the intestinal flora of the golden takin might change in the spring to enhance resistance and facilitate the ability to withstand changes in the outside world.

Previous researches also showed that the fecal microbiota of many mammals might be influenced by sex (Bian et al. 2013; Liu et al. 2014; Wu et al. 2016). In present study, the four female golden takins demonstrated highly similar microbiota and the five golden takin males had relatively highly similar microbiota, although $\mathrm{J} 6$ and $\mathrm{J} 7$ were relatively far from the other three samples, which might indicate that the fecal microbiota of golden takins might also be influenced by the animal's sex. Sex-related differences might be induced by sex hormones and their effects on gene expression as well as the immune system but also might be attributable to innate physiological differences between males and females (Mcclelland and Smith 2011; Zhao et al. 2013). However, the physiological mechanisms underlying sexual influence were uncertain.

In conclusion, we describe the predominant fecal bacterial populations in golden takin and offered the taxonomic baseline for further research investigating the intestinal ecosystems in golden takins. We also found that the composition of gut microbiota was influenced by the season which might help to strengthen the management of feed quality in the spring to prevent disease in these animals. These observations also increased our understanding of the bacterial ecosystems in this vulnerable animal.

\section{Abbreviations}

DNA: deoxyribonucleic acid; PCR: polymerase chain reaction; PCA: principal component analysis; OTUs: operational taxonomic unit.

\section{Authors' contributions}

Conceived and designed the experiments: HHZ, JC. Performed the experiments: JC, XYW. Analyzed the date: JC, HXZ. Contributed materials: JC, JKY, SS, YC. Wrote the paper: JC. Modify the manuscript: XXT. All authors read and approved the final manuscript.

\section{Acknowledgements}

We thank Ying Gao (Jinan Wild Animal Park), Xuchang Xie (Jinan Animal Park) for their assistance in the fecal samples collection.

\section{Competing interests}

The authors declare that they have no competing interests.

\section{Availability of date and materials}

The raw data obtained had been deposited in the NCBI Sequence Read Archive (SRA) with the Bio Project ID- PRJNA344965, Accession: SRP090712, ID: 090712.

\section{Ethical approval and consent to participate}

All applicable international, national, and/or institutional guidelines for the care and use of animals were followed.

\section{Funding}

This work was supported in part by grants from the Special Fund for Forest Scientific Research in the Public Welfare (No. 201404420), the National Natural Science Fund of China (Nos. 31372220, 31672313), Science and technology research plan of Shandong Province (No. 2013GSF11707).

\section{Publisher's Note}

Springer Nature remains neutral with regard to jurisdictional claims in published maps and institutional affiliations.

Received: 5 January 2017 Accepted: 21 March 2017

Published online: 17 April 2017

\section{References}

Bhatt VD, Dande SS, Patil NV, Joshi CG (2013) Molecular analysis of the bacterial microbiome in the forestomach fluid from the dromedary camel (Camelus dromedarius). Mol Biol Rep 40(4):3363-3371

Bian G, Ma L, Su Y, Zhu W (2013) The microbial community in the feces of the white rhinoceros (Ceratotherium simum) as determined by barcoded pyrosequencing analysis. PLOS ONE 8(7):e70103-e70103

Bull AT, Stach JEM, Ward AC, Goodfellow M (2005) Marine actinobacteria: perspectives, challenges, future directions. Antonie Van Leeuwenhoek $87(1): 65-79$

Cadwell K (2014) Expanding the role of the virome: commensalism in the gut. J Virol 89(4):1951-1953

Caporaso JG, Kuczynski J, Stombaugh J, Bittinger K, Bushman FD, Costello EK, Fierer N, Peña AG, Goodrich JK, Gordon JI (2010) QIIME allows analysis of high-throughput community sequencing data. Nat Methods 7(5):335-336

Carey HV, Walters WA, Knight R (2012) Seasonal restructuring of the ground squirrel gut microbiota over the annual hibernation cycle. Am J Physiol Regul Integr Comp Physiol 304(1):R33-R42

Costa MC, Arroyo LG, Allenvercoe E, Stämpfli HR, Kim PT, Sturgeon A, Weese JS (2012) Comparison of the fecal microbiota of healthy horses and horses 
with colitis by high throughput sequencing of the V3-V5 region of the 16S rRNA gene. PLoS ONE 7(7):267-278

Cundliffe $E$ (2006) Antibiotic production by actinomycetes: the Janus faces of regulation. J Ind Microbiol Biotechnol 33(7):500-506

Desantis TZ, Hugenholtz P, Larsen N, Rojas M, Brodie EL, Keller K, Huber T, Dalevi D, Hu P, Andersen GL (2006) Greengenes, a chimera-checked $16 \mathrm{~S}$ rRNA gene database and workbench compatible with ARB. Appl Environ Microbiol 72(7):5069-5072

Edgar RC (2004) MUSCLE: multiple sequence alignment with high accuracy and high throughput. Nucleic Acids Res 32(5):1792-1797

Edgar RC (2013) UPARSE: highly accurate OTU sequences from microbial amplicon reads. Nat Methods 10(10):996-998

Edgar RC, Haas BJ, Clemente JC, Quince C, Knight R (2011) UCHIME improves sensitivity and speed of chimera detection. Bioinformatics 27(16):2194-2200

Evans NJ, Brown JM, Murray RD, Getty B, Birtles RJ, Hart CA, Carter SD (2010) Characterization of novel bovine gastrointestinal tract Treponema isolates and comparison with bovine digital dermatitis treponemes. Appl Environ Microbiol 77(1):138-147

Hugerth LW, Wefer HA, Lundin S, Jakobsson HE, Lindberg M, Rodin S, Engstrand L, Andersson AF (2014) DegePrime, a program for degenerate primer design for broad-taxonomic-range PCR in microbial ecology studies. Appl Environ Microbiol 80(16):5116-5123

Jami E, Mizrahi I (2012) Composition and similarity of bovine rumen microbiota across individual animals. PLoS ONE 7(3):e33306

Kanokratana P, Uengwetwanit T, Rattanachomsri U, Bunterngsook B, Nimchua T, Tangphatsornruang S, Plengvidhya V, Champreda V, Eurwilaichitr L (2011) Insights into the phylogeny and metabolic potential of a primary tropical peat swamp forest microbial community by metagenomic analysis. Microb Ecol 61(3):518-528

Kim YS, Milner JA (2007) Dietary modulation of colon cancer risk. J Nutr 137(11 Suppl):2576S-2579S

Ley RE, Hamady M, Lozupone C, Turnbaugh PJ, Ramey RR, Bircher IS, Schlegel ML, Tucker TA, Schrenzel MD, Knight R (2008) Evolution of mammals and their gut microbes. Science 320(5883):1647-1651

Liu X, Fan H, Ding X, Hong Z, Nei Y, Liu Z, Li G, Guo H (2014) Analysis of the gut microbiota by high-throughput sequencing of the $\mathrm{V} 5-\mathrm{V} 6$ regions of the 16S rRNA gene in donkey. Curr Microbiol 68(5):657-662

Magoč T, Salzberg SL (2011) FLASH: fast length adjustment of short reads to improve genome assemblies. Bioinformatics 27(21):2957-2963

Mcclelland EE, Smith JM (2011) Gender specific differences in the immune response to infection. Arch Immunol Et Ther Exp 59(3):203-213

Ming L, Wei F, Groves P, Feng Z, Hu J (2003) Genetic structure and phylogeography of the takin (Budorcas taxicolor). Can J Zool 81(3):462-468(7)
Oikonomou G, Teixeira AG, Foditsch C, Bicalho ML, Machado VS, Bicalho RC (2013) Fecal microbial diversity in pre-weaned dairy calves as described by pyrosequencing of metagenomic $16 \mathrm{~S}$ rDNA. Associations of Faecalibacterium species with health and growth. PLOS ONE 8(4):7377-7382

Pankratov TA, Ivanova AO, Dedysh SN, Liesack W (2011) Bacterial populations and environmental factors controlling cellulose degradation in an acidic Sphagnum peat. Environ Microbiol 13(7):1800-1814

Salyers AA, Vercellotti JR, West SE, Wilkins TD (1977) Fermentation of mucin and plant polysaccharides by strains of Bacteroides from the human colon. Appl Environ Microbiol 33(2):319-322

Schaller GB, Teng Q, Pan W, Qin Z, Wang X, Jinchu HU, Shen H (1986) Feeding behavior of Sichuan takin (Budorcas taxicolor). Mammalia 50(3):311-322

Smith CJ, Rocha ER, Paster BJ (2006) The medically important bacteroides spp. in health and disease. In: Dworkin M, Falkow S, Rosenberg E, Schleifer K-H, Stackebrandt E (eds) The prokaryotes:volume 7: proteobacteria: delta, epsilon subclass. Springer, New York, pp 381-427. doi:10.1007/0-387-30747-8_14

Sun B et al (2016) Marked variation between winter and spring gut microbiota in free-ranging Tibetan Macaques (Macaca thibetana). Sci Rep 6:26035. doi:10.1038/srep26035

Taglialatela JP, Russell JL, Pope SM, Morton T, Bogart S, Reamer LA, Schapiro SJ, Hopkins WD (2009) A core gut microbiome in obese and lean twins. Nature 457(7228):480-484

Thoetkiattikul H, Mhuantong W, Laothanachareon T, Tangphatsornruang S, Pattarajinda V, Eurwilaichitr L, Champreda V (2013) Comparative analysis of microbial profiles in cow rumen fed with different dietary fiber by tagged 16S rRNA gene pyrosequencing. Curr Microbiol 67(2):130-137

Wang M, Ahrné S, Jeppsson B, Molin G (2005) Comparison of bacterial diversity along the human intestinal tract by direct cloning and sequencing of $16 \mathrm{~S}$ rRNA genes. FEMS Microbiol Ecol 54(2):219-231

Wang Q, Garrity GM, Tiedje JM, Cole JR (2007) Naive Bayesian classifier for rapid assignment of rRNA sequences into the new bacterial taxonomy. Appl Environ Microbiol 73(16):5261-5267

Wei F, Fang Z, Xiao Y (2012) Evidence for lignin oxidation by the giant panda fecal microbiome. PLoS ONE 7(11):e50312

Wu X, Zhang H, Chen J, Shang S, Wei Q, Yan J, Tu X (2016) Comparison of the fecal microbiota of dholes high-throughput Illumina sequencing of the V3-V4 region of the 16S rRNA gene. Appl Microbiol Biotechnol 100(8):1-10

Zhao L, Wang G, Siegel P, He C, Wang H, Zhao W, Zhai Z, Tian F, Zhao J, Zhang H (2013) Quantitative genetic background of the host influences gut microbiomes in chickens. Sci Rep 3(5):1970

\section{Submit your manuscript to a SpringerOpen ${ }^{\circ}$ journal and benefit from:}

- Convenient online submission

- Rigorous peer review

- Immediate publication on acceptance

- Open access: articles freely available online

- High visibility within the field

- Retaining the copyright to your article

Submit your next manuscript at $\boldsymbol{\nabla}$ springeropen.com 